\title{
NONLINEAR MODELING AND ANALYSIS OF A SHOCK ABSORBER WITH A BYPASS
}

\author{
Urszula Ferdek, Jan Łuczko \\ Cracow University of Technology, Faculty of Mechanical Engineering, Kraków, Poland \\ e-mail: uferdek@mech.pk.edu.pl; jluczko@mech.pk.edu.pl
}

\begin{abstract}
The model of a mono-tube shock absorber with a bypass is proposed in this paper. It is shown that the application of an additional flow passage (bypass) causes changes to the damping force characteristics when the excitation amplitudes are large. In such cases, the damping force values increase, thereby improving safety of the ride. For small excitation amplitudes, the shock absorber behaves in a similar fashion as shock absorbers without a bypass, ensuring a high comfort level of the ride on roads with smooth surfaces.
\end{abstract}

Keywords: shock absorber, hydraulic damper, vehicle suspension, nonlinear, vibrations

\section{Introduction}

The problems with the modelling and analysis of hydraulic dampers are discussed in several papers. It results from the fact of their wide applications (especially in the automotive industry) as well as from frequent employement of new damper designs (Norgaard and Cimins, 2009; King, 2014; Marking, 2014). These new concepts, often intuitively introduced, require successive theoretical solutions. One of such ideas is the application of an additional flow passage, meaning bypasses. Such solutions are introduced in order to improve safety of the ride, mainly in cross-country vehicles. When such vehicles run onto a large obstacle, the oil flow through a bypass becomes blocked off. As a result, the damping force increases suddenly, changing the damper characteristics. The constructional parameters of the damper with a bypass should be selected in such a way as to have - during travelling on smooth surfaces - the characteristic not worse than the characteristic of a hydraulic damper without a bypass.

Good comfortable rides are provided by dampers of 'soft' characteristics while dampers of 'hard' characteristics increase the safety, assuring better control and higher braking forces. In order to bring together these contradictory requirements, semi-active systems (Ferdek and Łuczko, 2015, 2016) usually magneto-rheological (Sapiński and Rosół, 2007; Gołdasz, 2015) are often applied. Compared to passive systems, semi-active dampers provide the possibility of adjusting the damping force to specific conditions of the ride. However, they have more complicated construction and due to that, they are more expensive both in production and in operations.

Currently used dampers have a flow passage which can be situated inside the piston rod (King, 2014) or outside the working cylinder (Norgaard and Cimins, 2009; Marking, 2014). Such bypasses can have an additional pressure valve controlling the oil flow. The application of the bypass changes the dampers characteristics. When a vehicle is going on relatively smooth surfaces, the displacement of the piston rod is small, and the oil flow between the chambers occurs both through the piston bleed orifices and through the bypass. This in turn creates a soft damper characteristic ensuring comfort of the ride. When the vehicle has to clear large obstacles, it means large piston strokes, the oil is not flowing through the bypass, and the damping force significantly increases ('hard' characteristic). 
The analysis of the car model requires the introduction of a relatively simple hydraulic damper model properly describing its basic properties and allowing simultaneously the investigation of the influence of essential parameters within the wide range of their changes. Tests of modelling twin-tube dampers (Ramos et al., 2005; Alonso and Comas, 2006), mono-tube dampers (Talbott and Starkey, 2002; Titurus et al., 2010; Farjoud et al., 2012) and others have been undertaken. They mainly differ in the approach to describing the oil flow through valves. Alonso and Comas (2006) investigated the twin-tube damper model taking into account the cavitation problem and the damper chambers elasticity. Talbott and Starkey (2002) investigated the mono-tube damper modelling the influence of the shim stack by the preliminarily pressed spring. They assumed that the laminar oil flow was a result of leakage in the piston-cylinder system, in contrast to the turbulent flow through the orifice system in the piston. Farjoud et al. (2012) investigated the influence of the shim stack properties on characteristics of the mono-tube damper. The authors compared the obtained results with the experimental ones. In the paper by Czop and Sławik (2011), the model of the twin-tube shock absorber was tested and experimentally verified.

There is a separate group of research papers dealing with the cavitation problem being a result of sudden oil pressure changes in hydraulic dampers. In the papers by Cho et al. (2002), Van de Ven (2013) and by Manring (1997) various descriptions of the effective bulk modulus were given.

Papers dealing with modelling of dampers with a bypass are relatively rare. Apart from patents (Norgaard and Cimins, 2009; $K_{i} \mathrm{ng}$, 2014; Marking, 2014), only in the paper by Lee and Moon (2006) the model of the displacement-sensitive shock absorber was discussed. Depending on the piston displacement, the flow control was realised by a proper configuration of the inner cylinder surface.

The purpose of the hereby paper is to demonstrate that the introducing of an additional flow passage to a classical hydraulic damper causes a change of the damping force characteristics. In the case of high excitation amplitudes, an increased value of the damping force improves the safety of the ride. While in the case of small excitation amplitudes, the damper behaves in a similar fashion as the classical shock absorber, where an increased oil flow causes a decrease in the damping force providing higher riding comfort. To assure the proper functioning of the model within the wide range of amplitudes and excitation frequencies, the pressure influence on the oil compressibility modulus is taken into account. The proposed model of the damper is different than the presented in the paper by Lee and Moon (2006) and allows the investigation of the influence of a more number of constructional parameters of the shock absorber within the wide range of their changes.

\section{Model of a variable damping shock absorber}

The scheme of the hydraulic shock absorber with a bypass as well as its model is presented in Fig. 1. Two chambers are in the main cylinder: chamber $K_{1}$ above the piston (rebound chamber) and chamber $K_{2}$ below the piston (compression chamber). Narrow orifices through which oil flows between both chambers are inside the piston. Some orifices are constantly open while the others are the most often covered by the shim stack. An additional external flow passage connects chambers $K_{1}$ and $K_{2}$, and distances $h_{1}$ and $h_{2}$ determine placements of the bypass orifices. The shock absorber is rigidly connected with a reserve cylinder, consisting of chamber $K_{3}$ filled with oil and chamber $K_{4}$ filled with gas under a high pressure of 2-3 MPa. The floating piston of a relatively small mass separates both chambers. Two phases of the piston rod motion are essential in the damper operations: the compression phase and rebound (expansion) phase. During the compression, the piston rod is moving down causing the pressure increase in 
chamber $K_{2}$ and the oil flow into chambers $K_{1}$ and $K_{3}$. During the rebound process, due to the pressure increase in chamber $K_{1}$, the oil returns to chamber $K_{2}$.
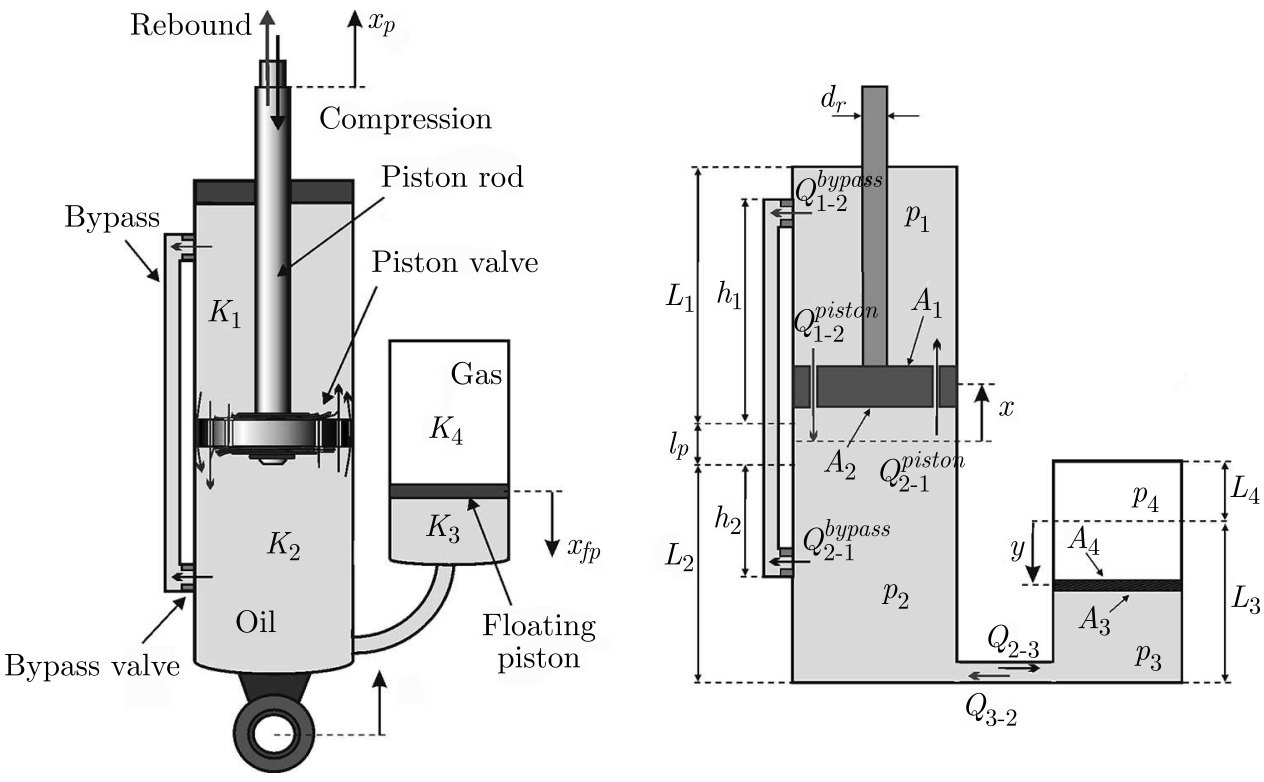

Fig. 1. The scheme and the model of the damper with a bypass

In the case of minor displacements and pressures, the oil flows only through bleed orifices not covered by plates through leakages and, eventually, through the bypass if the pressure controlled valve is not installed. Along with the pressure increase, the valves in the piston and bypass are gradually opened. During the rebound phase, the oil flows through differently designed piston orifices (of a different cross-sectional area) than in the compression phase, which - finally - causes the damper characteristic asymmetry. Asymmetrical characteristics of the shock absorber are desirable for comfort of the passengers (Silveira et al., 2014). When the piston exceeds the distance $h_{1}$ (during compression) or $h_{2}$ (during rebound), the proper entrance to the bypass becomes blocked, and the oil flows only through bleed orifices in the main piston.

The oil flow from chamber $K_{2}$ to $K_{3}$ occurs through a relatively short and stiff conduit of a significant cross-section. Coordinate $x_{p}$ determines the piston motion, $x_{c}$ - motions of both cylinders, while $x_{f p}$ - the floating piston motion. The relative displacements of corresponding pistons are determined by coordinates $x=x_{p}-x_{c}$ and $y=x_{f p}-x_{c}$. The motion of both pistons is measured from the static equilibrium position. Notation $p_{i}$ is used for pressures in chambers $K_{i}(i=1, \ldots, 4), A_{i}$ - for surfaces of the main and floating pistons $\left(A_{3}=A_{4}\right)$ and $V_{i}$ - for volumes of chambers $K_{i}$.

The resistance force depends mainly on the resultant pressure force acting on the piston, it corresponds to the oil pressures $p_{1}$ and $p_{2}$ in chambers $K_{1}$ and $K_{2}$. Taking into consideration the Coulomb friction force $F_{f 1}$ (Lee and Moon, 2006; Farjoud et al., 2012; Gołdasz, 2015) between the piston rod and the main cylinder, the damping force can be described as

$$
F=\left(p_{1}-p_{0}\right) A_{1}-\left(p_{2}-p_{0}\right) A_{2}+F_{f 1} \operatorname{sgn} \dot{x}
$$

where $p_{0}$ is the nominal working pressure. In the simulations, the signum function is approximated (Czop and Sławik, 2011) as follows: $\operatorname{sgn} \dot{x}=\tanh \left(\dot{x} /\right.$ vref), where $v_{\text {ref }}$ is the reference velocity value (in simulations $v_{r e f}=0.005 \mathrm{~m} / \mathrm{s}$ ). In order to determine the shock absorber characteristics, the most often a harmonic excitation is assumed in the form: $x(t)=a \sin \omega t$, where $a$ and $\omega$ are the amplitude and frequency of the excitation.

In order to determine pressures $p_{1}$ and $p_{2}$, the processes occurring in the chambers should be considered with a special attention directed to the proper description of the oil flows between 
the chambers. It will be assumed that the fluid is compressible, taking into account changes of the bulk modulus, especially in the low pressures range. The equation

$$
\frac{d \rho_{i}}{d p_{i}}=\frac{1}{\beta_{i}} \rho_{i}
$$

describes the oil density change $\rho_{i}$ in chamber $K_{i}$ caused by the pressure change $p_{i}(i=1,2,3)$. It is usually assumed that the bulk modulus value $\beta_{i}$ is constant $\left(\beta_{i}=\beta\right.$, where $\beta$ is the bulk modulus for the given pressure value, e.g. for the working pressure). This assumption is justified within the limited pressure changes, i.e. in a limited range of the amplitude and piston velocity. For large displacements and velocities, the pressure in one chamber significantly increases while in the other decreases. The assumption of the constant value of the bulk modulus can lead to physically inadmissible solutions of the analysed equations, sometimes even to negative pressure values. In reality, the bulk modulus for large pressures insignificantly increases, and for very small pressures the cavitation effect occurs, during which - due to liquid evaporation - gas bubbles are formed. The accurate description of this phenomenon is more complex and depends on several other factors. The effect of cavitation is a sudden compressibility increase; meaning a bulk modulus decrease. Cho et al. (2002) proposed different descriptions of the bulk modulus depending on the pressure and the oil aeration degree. Comparisons with experimental results were also presented. Van de Ven (2013) provided selected equations for the effective bulk modulus $\beta_{e}$. The simplest equation proposed by Merritt (1967) is of the following form

$$
\beta_{e}=\beta \frac{1}{R \beta / \kappa p+1}
$$

where $R$ is the volume fraction of the air at the atmospheric pressure $p_{a}$, while $\kappa$ is the adiabatic index. Hayward provides a slightly different equation

$$
\beta_{e}=\beta \frac{R+\bar{p}^{\kappa}}{R \beta / \kappa p+\bar{p}^{\kappa}}
$$

where $\bar{p}=p / p_{a}$. Another equation was proposed by Cho et al. (2002)

$$
\beta_{e}=\beta \frac{R+\bar{p}^{\kappa} \exp \left[\left(p_{a}-p\right) / \beta\right]}{R \beta / \kappa p+\bar{p}^{\kappa} \exp \left[\left(p_{a}-p\right) / \beta\right]}
$$

The shock absorber of properly selected parameters operates within the range of high pressures $(\sim 2 \mathrm{MPa})$, and then the compressibility modulus changes only insignificantly. However, in the designing process, the damper parameters can be changing in wide ranges. When the parameters are incorrectly selected, a malfunction of the shock absorber - manifested by large pressure changes - can occur. In such cases, there is a necessity of applying the proper equation for the effective bulk modulus.

The following one-parameter model is proposed in the hereby paper

$$
\beta_{e}(p)=\beta \tanh \frac{p}{p_{s}}
$$

Along with decreasing of the parameter $p_{s}$ value (reference pressure) the bulk modulus faster obtains the limit value $\beta$. Figure 2 presents comparisons of the effective bulk modulus diagrams obtained for models: (2.3)-(2.6), for two relatively small values of the parameter $R$. For the given values of the parameter $p_{s}$, the proposed model (2.6) indicates the best compatibility with model (2.4). Within the working pressures range $\left(p_{0}=2 \mathrm{MPa}\right)$ the bulk modulus is close to $\beta$, and for low pressures it fastly approaches zero. The advantage of formula (2.6) constitutes the possibility 

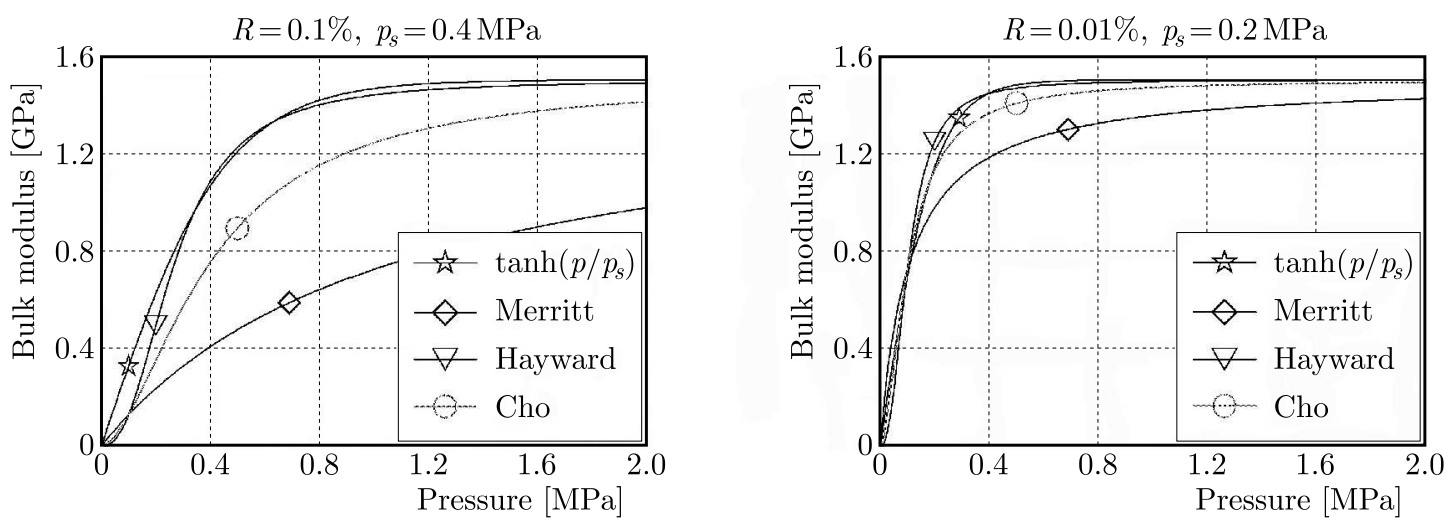

Fig. 2. Bulk modulus versus pressure for different values of $R$ and $p_{s}$

of obtaining an analytical equation describing the oil density. After substituting formula (2.6) into equation (2.2), the following expression is obtained

$$
\rho_{i}=\rho_{0}\left[\frac{\sinh \left(p_{i} / p_{s}\right)}{\sinh \left(p_{0} / p_{s}\right)}\right]^{p_{s} / \beta}
$$

where $\rho_{0}$ is the oil density under the working pressure $p_{0}$.

In order to determine the oil pressure in chambers $K_{i}$, equations of the general form can be used

$$
\dot{\rho}_{i} V_{i}+\rho_{i} \dot{V}_{i}=Q_{i}
$$

where $Q_{i}=\dot{m}_{i}$ are mass flow rates. Volumes of chambers $K_{i}(i=1,2,3)$ can be calculated as follows

$$
V_{1}=A_{1}\left(L_{1}-x\right) \quad V_{2}=A_{2}\left(L_{2}+x\right) \quad V_{3}=A_{3}\left(L_{3}-y\right)
$$

where distances $L_{1}$ and $L_{2}$ are lengths of chambers $K_{1}$ and $K_{2}$ in the working cylinder for $x=0$, while $L_{3}$ and $L_{4}$ are lengths of chambers $K_{3}$ and $K_{4}$ in the external cylinder for $y=0$. The relative displacement $y$ of the floating piston can be determined from the differential equation

$$
m_{f p} \ddot{y}=\left(p_{4}-p_{3}\right) A_{3}-F_{f 2} \operatorname{sgn} \dot{y}
$$

where $m_{f p}$ is the floating piston mass, and $F_{f 2}$ is the friction force between this floating piston and the reserve cylinder. Gas pressure $p_{4}$ is determined from the equation of the polytropic process: $p_{4} V_{4}^{n}=p_{0} V_{40}^{n}$ (Farjoud et al., 2012; Ferdek and Euczko, 2012), where: $V_{4}=A_{4}\left(L_{4}+y\right)$ and $V_{40}=A_{4} L_{4}$. Hence, it follows

$$
p_{4}=p_{0} \frac{L_{4}^{n}}{\left(L_{4}+y\right)^{n}}
$$

After using equation (2.2) and transforming equations (2.8), the equations describing the oil pressures in the chambers $K_{i}(i=1,2,3)$ take the form

$$
\dot{p}_{1}=\frac{\beta_{1}}{V_{1}}\left(\frac{Q_{1}}{\rho_{1}}+A_{1} \dot{x}\right) \quad \dot{p}_{2}=\frac{\beta_{2}}{V_{2}}\left(\frac{Q_{2}}{\rho_{2}}-A_{2} \dot{x}\right) \quad \dot{p}_{3}=\frac{\beta_{3}}{V_{3}}\left(\frac{Q_{3}}{\rho_{3}}+A_{3} \dot{y}\right)
$$

where the moduli $\beta_{i}=\beta_{e}\left(p_{i}\right)$ in the respective chambers are determined by formula (2.6) and the densities by (2.7). System (2.12) of non-linear differential equations of the first order and differential equation (2.10) of the second order constitute the base for the determination of characteristic (2.1) of the mono-tube hydraulic shock absorber with and without the bypass. 

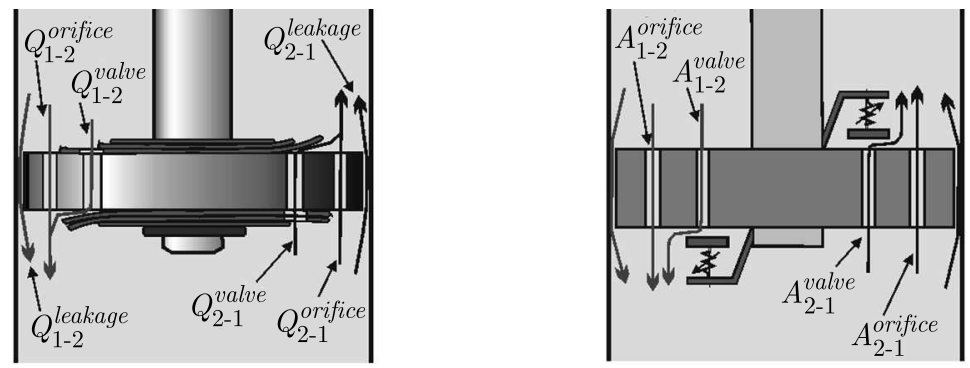

Fig. 3. Diagram showing the flow paths

One can denote $Q_{j-i}(j=1, i=2$ or $j=2, i=1)$ - the mass flow rate from chamber $K_{j}$ (e.g. rebound for $j=1$ ) to chamber $K_{i}$ (e.g. compression $i=2$ ). In the case of the flow in the reverse direction: $Q_{j-i}=0$ (then $Q_{i-j} \neq 0$ ). Since the oil flow between these chambers occurs through orifices in the piston (Fig. 3) and through the bypass, the flow rate can be written as

$$
Q_{j-i}=Q_{j-i}^{\text {piston }}+Q_{j-i}^{\text {bypass }}
$$

where the flow rate $Q_{j-i}^{\text {piston }}$ is the sum of three flow rates (Fig. 3)

$$
Q_{j-i}^{\text {piston }}=Q_{j-i}^{\text {leakage }}+Q_{j-i}^{\text {orifice }}+Q_{j-i}^{\text {valve }}
$$

representing the flow rates resulting from leakage past piston, flow through bleed orifices and flow through valves in the piston. The oil flow in the reversed direction, from chamber $K_{i}$ to $K_{j}$, determined by the mass flow rate $Q_{i-j}$, causes a mass decrease in chamber $K_{i}$. Thus, the oil mass change in chamber $K_{i}$ can be written in the following form

$$
Q_{i}=Q_{j-i}-Q_{i-j}
$$

Since, from the law of mass conservation between mass flow rates the following relation occurs: $Q_{1}+Q_{2}+Q_{3}=0$. It is enough to determine the mass flow rates $Q_{1}$ and $Q_{3}$ determining mass changes in chambers $K_{1}$ and $K_{3}$. It results that $Q_{2}=-Q_{1}-Q_{3}$. The negative value of $Q_{i}$ is related to the oil outflow from chamber $K_{i}$.

Assuming the laminar flow (Talbott and Starkey, 2002), the mass flow rate $Q_{2}$-1leakage from the compression chamber to the rebound chamber can be determined from the equation

$$
Q_{2-1}^{\text {leakage }}=\pi d_{p}\left(\frac{b_{p c}^{3}\left(p_{2}-p_{1}\right)}{12 l_{p} \nu}-\frac{\rho_{2} b_{p c} \dot{x}}{2}\right)
$$

where $b_{p c}$ is clearance, $d_{p}$ - piston diameter, $l_{p}$ - piston length, $\nu$ - coefficient of kinematic viscosity. The first component of equation (2.16) determines the flow rate caused by the pressure difference while the second by the relative piston velocity. In the case when the piston moves up (for $\dot{x}>0$ ), the flow rate caused by the pressure difference is decreasing. Equation (2.16) is correct only for $Q_{2-1}^{\text {leakage }}>0$. Otherwise, the flow rate is determined from the following equation

$$
Q_{1-2}^{\text {leakage }}=\pi d_{p}\left(\frac{b_{p c}^{3}\left(p_{1}-p_{2}\right)}{12 l_{p} \nu}+\frac{\rho_{1} b_{p c} \dot{x}}{2}\right)
$$

In order to determine the remaining component of equation (2.11) for the turbulent flow (Titurus et al., 2010), the equation of a general form is used

$$
Q_{j-i}=C_{d} A_{j-i} \sqrt{2 \rho_{j}\left(p_{j}-p_{i}\right)}
$$


where $C_{d}$ is the discharge coefficient while $A_{j-i}$ is the effective cross-sectional area of the proper orifice through which the oil flows from chamber $K_{j}$ to $K_{i}$. Equation (2.18) is correct for $p_{j}>p_{i}$. After introduction of the function

$$
\vartheta\left(p_{j}, p_{i}, \rho_{j}\right)=C_{d} H\left(p_{j}-p_{i}\right) \sqrt{2 \rho_{j}\left(p_{j}-p_{i}\right)}
$$

where $H(\cdot)$ is the unit step function, equation (2.13) obtains the form

$$
Q_{j-i}=Q_{j-i}^{\text {leakage }}+\left(A_{j-i}^{\text {orifice }}+A_{j-i}^{\text {valve }}+A_{j-i}^{\text {bypass }}\right) \vartheta\left(p_{j}, p_{i}, \rho_{j}\right)
$$

Out of parameters: $A_{j-i}^{\text {oriffice }}, A_{j-i}^{\text {valve }}, A_{j-i}^{\text {bypass }}$, determining the effective areas of respective orifices, only the parameter $A_{j-i}^{\text {oriffice }}$ is of a constant value. After referring the orifice area to the area of the compression side of the piston, this area depends on the dimensionless parameter $\alpha_{j-i}$ in the following way

$$
A_{j-i}^{\text {orifice }}=\alpha_{j-i} A_{2}
$$

Values of the remaining areas depend on the oil pressure in the neighbouring chambers of the shock absorber and on the relative piston displacement.

The flow through the compression intake or through the rebound intake is controlled by pressures $p_{1}$ and $p_{2}$. Bleed orifices are the most often covered by a stack of circular plates (Fig. 3) deflecting under the influence of the resultant pressure force, and gradually uncovering the orifices. The effective cross-sectional area depends mainly on the pressure difference $p_{1}-p_{2}$ in the shock absorber chambers as well as on geometrical and physical parameters of the plates. The accurate determination of the area change law requires the accurate modelling of the specific technical solution. Disregarding inertia of the plates, the valve can be modelled by means of a stiff plate preliminarily pressed down by a spring of a progressive characteristic. For minute pressure differences and until the resultant pressure force is lower than the preload force, the bleed orifice remains closed. Only after exceeding the preload force, the orifice is gradually uncovered. The parameter $A_{j-i}^{\text {valve }}$ (effective area) decides which flow depends on the spring deflection. It can not, however, exceed the total area of the orifice cross-section. A function $\theta_{1}$ will be used for description of a change in the area. This function is defined as follows

$$
\theta_{1}\left(p_{j}-p_{i}, \sigma, k\right)=H\left(p_{j}-p_{i}-\sigma\right) \tanh \frac{p_{j}-p_{i}-\sigma}{k}
$$

where the parameter $\sigma$ determines the pressure difference value above which the plates start to deflect uncovering the bleed orifice joining the neighbouring chambers $\left(\theta_{1} \neq 0\right.$ only for $p_{j}-p_{i}>\sigma$ ), while the parameter $k$ characterises elastic properties of the shim stack. Using the hyperbolic tangent function in equation (2.22) ensures that the effective area $A_{j-i}^{\text {valve }}$ will not exceed the area of the orifice cross-section. The efficient (variable) valve area, it means the parameter $A_{j-i}^{\text {valve }}$, is determined by

$$
A_{j-i}^{\text {valve }}=\delta_{j-i} A_{2} \theta_{1}\left(p_{j}-p_{i}, \sigma^{\text {valve }}, k^{\text {valve }}\right)
$$

where the dimensionless parameter $\delta_{j-i}$ is the ratio of the orifice cross-sectional area to the area of the compression side of the piston. Values of dimensionless coefficients $\delta_{2-1}$ and $\delta_{1-2}$ (also $\alpha_{2-1}$ and $\alpha_{1-2}$ ), deciding respectively on flows in the compression and rebound process, can be different (the most often $\delta_{12}<\delta_{21}$ ) for ensuring a higher resistance force during rebound (for $\dot{x}>0$ ) in relation to the force created during the compression process (for $\dot{x}<0$ ).

The opening or closing of the bypass is controlled by the relative piston displacement $x$. The bypass whose openings are of a round cross-section of radius $r$ is gradually closed within 
the range $\left(h_{2}-r, h_{2}+r\right)$ in the rebound phase and within the range $\left(-h_{1}-r,-h_{1}+r\right)$ in the compression process. To describe the variable area $A_{j-i}^{\text {bypass }}$, the function defined below is suitable

$$
\theta_{2}\left(x, h_{1}, h_{2}, r\right)= \begin{cases}0 & \text { for } \quad x \geqslant h_{2}+r \\ \theta_{0}\left[\left(x-h_{2}\right) / r\right] & \text { for } \quad h_{2}-r<x<h_{2}+r \\ 1 & \text { for } \quad-h_{1}+r \leqslant x \leqslant h_{2}-r \\ \theta_{0}\left[\left(-x-h_{1}\right) / r\right] & \text { for } \quad-h_{1}-r<x<-h_{1}+r \\ 0 & \text { for } \quad x \leqslant-h_{1}-r\end{cases}
$$

where the function $\theta_{0}$ takes into account the round shape of the bypass orifices and is defined as

$$
\theta_{0}(\xi)=\frac{1}{\pi}\left(\arccos \xi-\xi \sqrt{1-\xi^{2}}\right)
$$

Assuming that the valve controlled by the pressure difference is additionally assembled in the bypass, after using functions (2.22) and (2.24), the effective bypass area is described by

$$
A_{j-i}^{\text {bypass }}=\gamma_{j-i} A_{2} \theta_{1}\left(p_{j}-p_{i}, \sigma^{\text {bypass }}, k^{\text {bypass }}\right) \theta_{2}\left(x, h_{1}, h_{2}, r\right)
$$

where $\gamma_{j-i} A_{2}$ determines the bypass cross-sectional area. The flow from the compression chamber $K_{2}$ to the reserve chamber $K_{3}$ can be described in a similar way. However, due to a significant diameter of the conduit which joing these chambers and the rarely used pressure valve, the mass flow rate is be described by the following equation

$$
Q_{j-i}=A_{j-i}^{\text {conduit }} \vartheta\left(p_{j}, p_{i}, \rho_{j}\right)
$$

where $j=2, i=3$ or $j=3, i=2$. The parameter $A_{2-3}^{\text {conduit }}=A_{3-2}^{\text {conduit }}$ describes the cross-sectional area of the conduit joining chambers $K_{2}$ and $K_{3}$.

\section{Effect of model parameters on the characteristics of the shock absorber}

The basic characteristics of the shock absorber are dependences of damping forces (2.1) on piston displacements and its relative velocity. The damping force depends mainly on the oil pressure in the compression and rebound chambers. The determination of characteristics requires integration of non-linear differential equations (2.10) and (2.12) describing the floating piston motion and the pressures. For the numerical integration, the Runge-Kutta method of the 5-th order has been used.

The values of the characteristic parameters of the shock absorber are given in Table 1 . The majority of these values results from the analysis of the existing structural solutions of mono-tube dampers. The values of the excitation parameters are changed within the specified ranges. The special attention is directed towards parameters influencing the mass flow rate and characterising the bypass. Apart from dimensional parameters given in Table 1, a significant influence on the solutions have dimensionless parameters $\alpha_{1-2}, \alpha_{2-1}, \delta_{1-2}, \delta_{2-1}, \gamma_{1-2}, \gamma_{2-1}$ characterising cross-section areas of the respective orifices. A number of these parameters can be limited by assuming that the areas of orifices - active in the rebound process - are proportional to the corresponding areas in the compression process. After introducing a coefficient $\lambda$ (in numerical calculations: $\lambda=0.6$ ), it can be assumed: $\alpha_{1-2}=\lambda \alpha, \alpha_{2-1}=\alpha, \delta_{1-2}=\lambda \delta, \delta_{2-1}=\delta$. It is also convenient to introduce dimensionless parameters $k_{p}$ and $k_{b}$ characterising the valve stiffness in the piston $\left(k^{\text {valve }}=k_{v} p_{0}\right)$ and in the bypass $\left(k^{\text {bypass }}=k_{b} p_{0}\right)$, respectively, as well as the dimensionless parameter $S_{v}$ related to the preload force in the piston $\left(\sigma^{v a l v e}=S_{v} p_{a}\right)$. Investigations of the bypass parameters influence are limited to the case of the symmetric distribution of bypass 
Table 1. Parameters of the shock absorber model

\begin{tabular}{|c|l|c|c|}
\hline Symbol & Description & Value & Unit \\
\hline \hline$A_{1}$ & Cross-sectional area of chamber $K_{1}$ & 24.6 & $\mathrm{~cm}^{2}$ \\
\hline$A_{i}$ & Cross-sectional area of chambers $K_{2}, K_{3}$ and $K_{4}$ & 28.3 & $\mathrm{~cm}^{2}$ \\
\hline$d_{p}$ & Diameter of piston & 6 & $\mathrm{~cm}$ \\
\hline$d_{r}$ & Diameter of piston rod & 2.2 & $\mathrm{~cm}$ \\
\hline$d_{c}$ & Diameter of conduit & 1 & $\mathrm{~cm}$ \\
\hline$l_{p}$ & Piston length & 1.5 & $\mathrm{~cm}$ \\
\hline$b_{p c}$ & Clearance & 0.1 & $\mathrm{~mm}$ \\
\hline$h$ & Distance between bypass orifices & $2-5$ & $\mathrm{~cm}$ \\
\hline$F_{f 1}$ & Friction force between piston and cylinder & 10 & $\mathrm{~N}$ \\
\hline$F_{f 2}$ & Friction between floating piston and oil tank & 1 & $\mathrm{~N}$ \\
\hline$m_{f p}$ & Floating piston mass & 0.1 & $\mathrm{~kg}$ \\
\hline$p_{0}$ & Nominal working pressure & 2 & $\mathrm{MPa}$ \\
\hline$p_{a}$ & Atmospheric pressure & 0.1 & $\mathrm{MPa}$ \\
\hline$C_{d}$ & Discharge coefficient & 0.6 & - \\
\hline$\beta$ & Fluid bulk modulus at atmospheric pressure & 1.5 & $\mathrm{GPa}$ \\
\hline$\beta$ & Kinematic viscosity of hydraulic oil & 32 & $\mathrm{cSt}$ \\
\hline$\beta$ & Oil density at atmospheric pressure & 890 & $\mathrm{~kg} / \mathrm{m}^{3}$ \\
\hline$a$ & Amplitude & $1-8$ & $\mathrm{~cm}$ \\
\hline$f$ & Excitation frequency & $1-12$ & $\mathrm{~Hz}$ \\
\hline
\end{tabular}

orifices $\left(h_{1}=h_{2}=h\right.$, and $\left.\gamma_{1-2}=\gamma_{2-1}=\gamma_{1}\right)$ and for the elastic valve without preliminary deflections $\left(\sigma^{\text {bypass }}=0\right)$.

The influence of damping forces on the piston relative displacement $x$ is presented in Fig. 4 . The curves illustrate the effect of the excitation amplitude $a$ (for $f=\omega / 2 \pi=1.4 \mathrm{~Hz}$ ) and the effect of frequency $f$ (for $a=3 \mathrm{~cm}$ ) on the damper characteristics. The assumed excitation frequency $f=1.4 \mathrm{~Hz}$ corresponds to the first frequency of natural vibrations in several models of vehicles. Excited vibrations, within the resonance range enclosing this frequency, worsen the ride comfort.
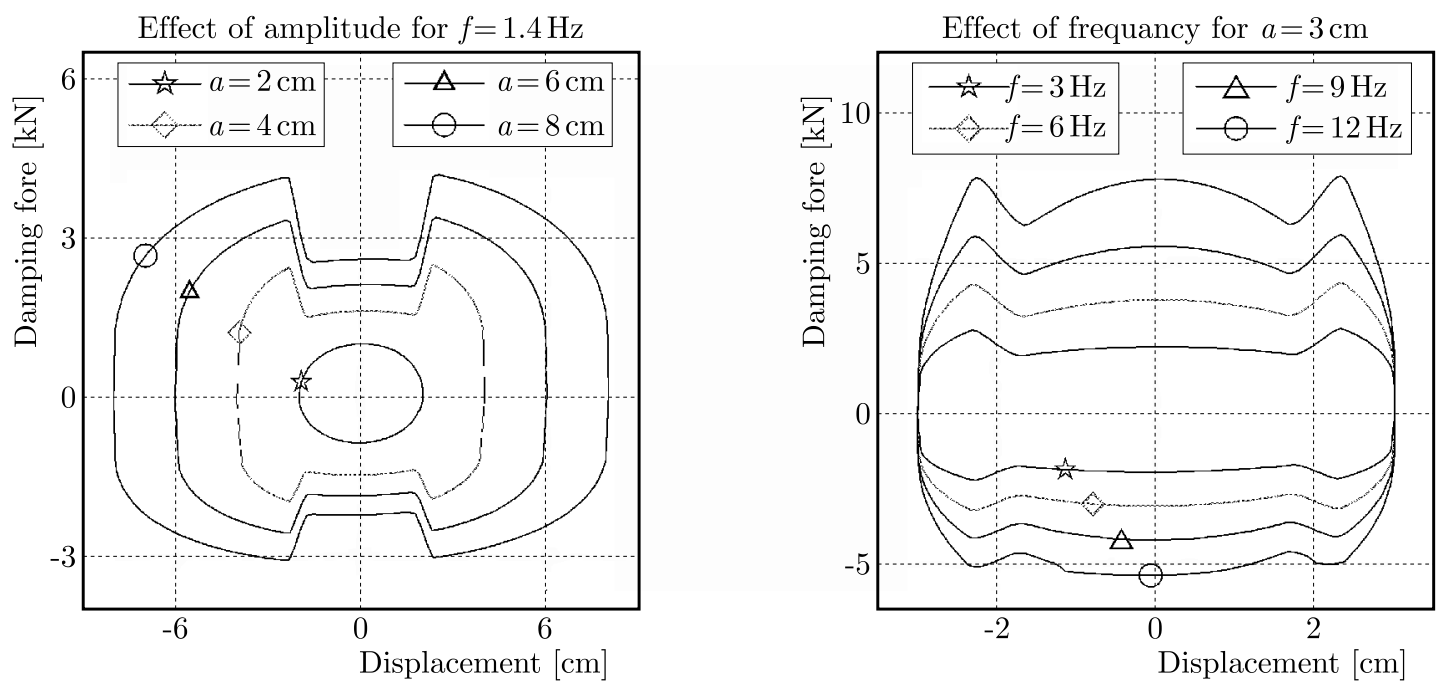

Fig. 4. Influence of the amplitude and excitation frequency on the damping force characteristics $\left(\alpha=0.004, \gamma=0.015, \delta=0.04, S_{v}=5, k_{p}=2, k_{b}=0.5, h=2 \mathrm{~cm}\right)$ 
The presented curves are determined for relatively large amplitude values. They are aimed at clear observation of the bypass influence. For the assumed parameter $h=2 \mathrm{~cm}$ the flow through the bypass is not blocked by the piston, only for the amplitude $a=2 \mathrm{~cm}$. However, along with an increase of the amplitude and the excitation frequency, the damping forces grow, but within the range of the active flow through the bypass they are significantly lower (for $-h+r<x<h-r)$. Only after surpassing this range, the damping force suddenly increases, which is caused by covering the openings of the additional orifice. Thus, within this range, the damper changes its properties and becomes the so-called 'hard' damper, which improves the safety level when a vehicle travels on surfaces with large irregularities. When a vehicle travels on good surfaces (for small amplitudes), the bypass is active all the time, and the damping force is not suddenly increasing.

The influence of the excitation amplitude on the damping force characteristics presented in Fig. 4 is qualitatively similar to the results of experiments discussed by Lee and Moon (2006).

The excitation frequency is related to the ride velocity, and this usually depends on the road class. When the damper performance is investigated within the high frequency range, respectively lower excitation amplitudes should be assumed, e.g. by limiting the maximum piston velocities. Characteristics of shock absorbers are the most often determined within the velocity range not exceeding $v_{0} \approx 1 \mathrm{~m} / \mathrm{s}$. In the case of harmonic motion, this leads to the condition: $2 \pi f a \leqslant v_{0}$. Out of all characteristics shown in Fig. 4, the curves obtained for $f=9 \mathrm{~Hz}$ and $f=12 \mathrm{~Hz}$ do not satisfy this condition. The effect of high piston velocities, especially for $f=12 \mathrm{~Hz}, a=3 \mathrm{~cm}$, constitute large changes of the oil pressure in the damper chambers. For the excitation parameters selected in such a way, the oil pressure in the rebound chamber during the compression phase suddenly decreases causing a significant decrease of the bulk modulus. The assumption of a constant bulk modulus, in this drastic case, would lead to a negative pressure $p_{1}$, physically unacceptable. Pressure values for the proposed description of the bulk modulus (2.6) are always positive. The excitation frequency $f=12 \mathrm{~Hz}$ in several vehicle models (Lee and Moon, 2006) is contained in the second resonance range, corresponding to higher velocities for which the excitation amplitudes satisfy the discussed above limitation. For such amplitudes (smaller than $2 \mathrm{~cm}$ ) the bypass is not blocked by the piston, and the damper characteristic is close to the 'soft' one.

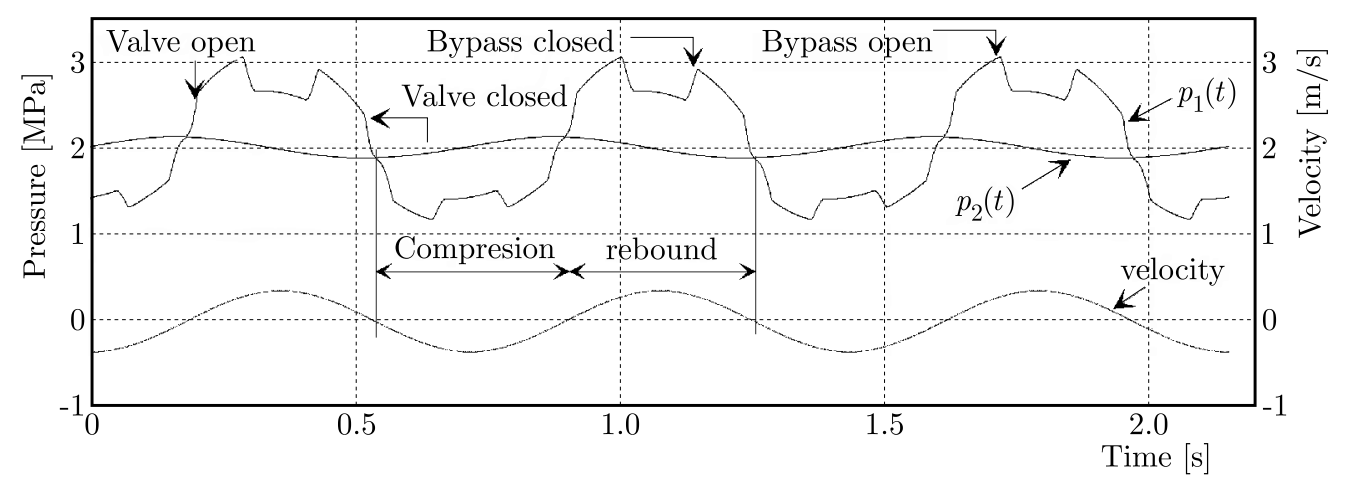

Fig. 5. Time histories of pressures $(f=1.4 \mathrm{~Hz}, a=4 \mathrm{~cm}, h=2 \mathrm{~cm}, \alpha=0.004, \gamma=0.015, \delta=0.04$,

$$
\left.S_{v}=5, k_{p}=2, k_{b}=0.5\right)
$$

The typical diagrams of pressure $p_{1}$ time waveforms in the rebound chamber $K_{1}$ and pressure $p_{2}$ in the compression chamber $K_{2}$ are shown in Fig. 5. Oil pressure $p_{3}$ and gas $p_{4}$ time histories in chambers $K_{3}$ and $K_{4}$ are similar to the pressure $p_{2}$ diagram. It is easy to determine time intervals corresponding to the compression and rebound, by analysing the sign of the relative piston velocity. Pressure $p_{1}$ in the rebound chamber has the decisive influence on the damping force. Changes of the remaining pressures are similar to the harmonic waveforms, which is the 
result of connecting the compression chamber with the reservoir of accumulative properties. When observing the pressure $p_{1}(t)$ diagram, the characteristic points in which pressure changes are either softer or more sudden can be noticed in the rebound and compression phases. This is the effect of opening or closing valves in the piston or bypass.

The functions of the mass flow rates on the relative piston displacement as well as diagrams of the effective (variable) areas deciding on flows through the corresponding valves are shown in Fig. 6. The shown functions: $Q^{\text {leakage }}=Q_{2-1}^{\text {leakage }}-Q_{1-2}^{\text {leakage }}, Q^{\text {oriffice }}=Q_{2-1}^{\text {oriffice }}-Q_{1-2}^{\text {oriffice }}$, $Q^{\text {valve }}=Q_{2-1}^{\text {valve }}-Q_{1-2}^{\text {valve }}, Q^{\text {bypass }}=Q_{2-1}^{\text {bypass }}-Q_{1-2}^{\text {bypass }}$ characterise the oil flow into the rebound chamber. Negative values of the mass flow rates are related to a mass decrease. It means that they correspond to the oil outflow from chamber $K_{1}$. Diagrams of the total flow rates $Q_{2-1}-Q_{1-2}$ and $Q_{2-3}-Q_{3-2}$ (mass change in chamber $K_{3}$ ) are also seen in Fig. 6. The respective areas (except areas characterising leakages) are defined in a similar fashion. Cyclic repetitions of curves pathways in Fig. 6 signify the motion periodicity.
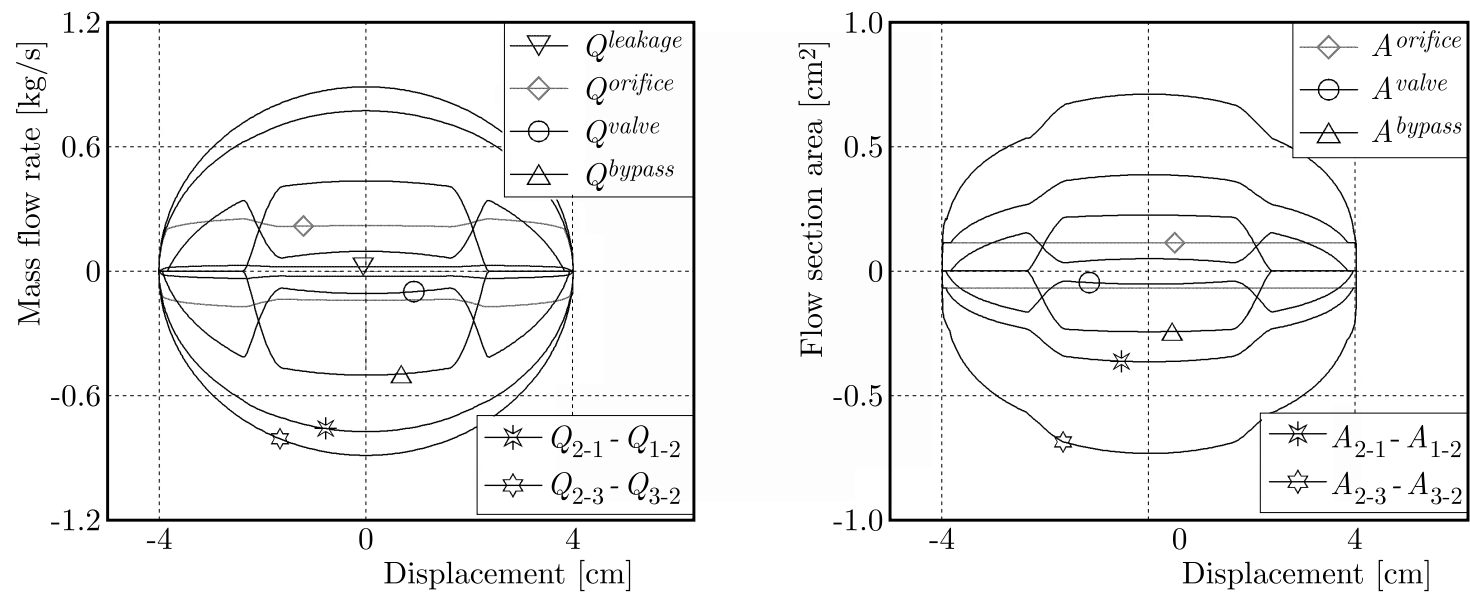

Fig. 6. Mass flow rates and effective areas $(f=1.4 \mathrm{~Hz}, a=4 \mathrm{~cm}, h=2 \mathrm{~cm}, \alpha=0.004, \gamma=0.015$, $\left.\delta=0.04, S_{v}=5, k_{p}=2, k_{b}=0.5\right)$

The presented diagrams allow one to better understand the oil flow processes between individual chambers of the damper. The most interesting of which are diagrams concerning flows through valves placed in the piston $\left(Q^{\text {valve }}, A^{\text {valve }}\right)$ and in the bypass $\left(Q^{\text {bypass }}, A^{\text {bypass }}\right)$. At the moment of bypass opening, the pressure $p_{1}$ suddenly decreases in the rebound chamber, which also causes the pressure difference to decrease in the neighbouring chambers. In effect, both the area $A^{\text {valve }}$ and the flow rate $Q^{\text {valve }}$ through the valve in the piston are thereby decreasing. For insignificantly higher values of the parameter $S_{v}$ (e.g. for $S_{v} \approx 7$ ), characterising the preload force, this valve is closed within small displacements. The fact that the total flow rates $Q_{2-1}$ and $Q_{2-3}$ are changing very regularly, regardless of values of the parameters characterising the valves, is very interesting.

The diagrams of the damping force versus displacement and the relative piston velocity are presented in Figs. 7-9. The shown characteristics successively illustrate the influence of the dimensionless parameters $\alpha, \delta$ and $S_{v}$, deciding on flows through the orifices placed in the piston. The force dependence on displacements is in all cases qualitatively similar to those discussed earlier. Much more interesting are diagrams of the damping force versus velocity. Inflection points determining the velocity ranges in which the characteristics have different waveforms are seen in the diagrams.

The parameter $\alpha$ determining the areas of constant orifices (it is assumed that $\alpha_{2-1}=\alpha$, $\alpha_{1-2}=\lambda \alpha$ ) is essential within the range of small velocities (Fig. 7). Along with the increasing parameter $\alpha$, the inclination angle of the curve $F(\dot{x})$ decreases. This means that the damping forces for the given velocity are smaller, which is beneficial from the point of view of the ride 

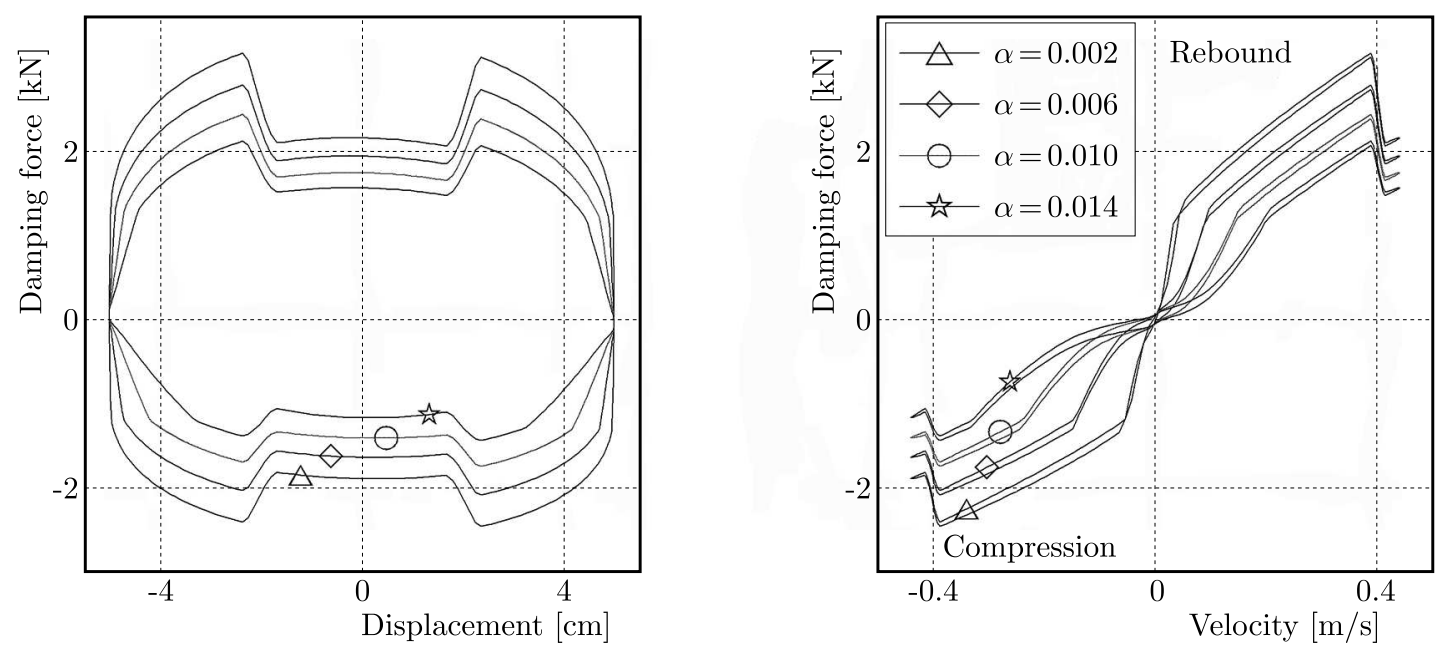

Fig. 7. Influence of the parameter $\alpha$ on the damping force characteristic $(f=1.4 \mathrm{~Hz}, a=5 \mathrm{~cm}$, $\left.h=2 \mathrm{~cm}, \delta=0.04, \gamma=0.012, S_{v}=5, k_{p}=2, k_{b}=0.5\right)$

comfort. In the diagrams of forces versus velocities, narrow hysteresis loops occur, which means that the damper behaves differently in positive and negative ranges of relative displacements. For the excitation frequency $f=1.4 \mathrm{~Hz}$ (within the first resonance range) the loop width is nearly constant in the whole velocity range. When the frequency increases, the loop width significantly increases for low piston velocities and decreases for higher velocities. Thus, the characteristics are non-symmetrical. This asymmetry results mainly from various areas of the orifices through which the oil flows in the compression and rebound processes. The parameter $\lambda$ decides about the model characteristic asymmetry. For $\lambda<1$ (in simulations $\lambda=0.6$ ), the damper offers higher resistances in the rebound process (for $\dot{x}>0$ ) than in the compression one.
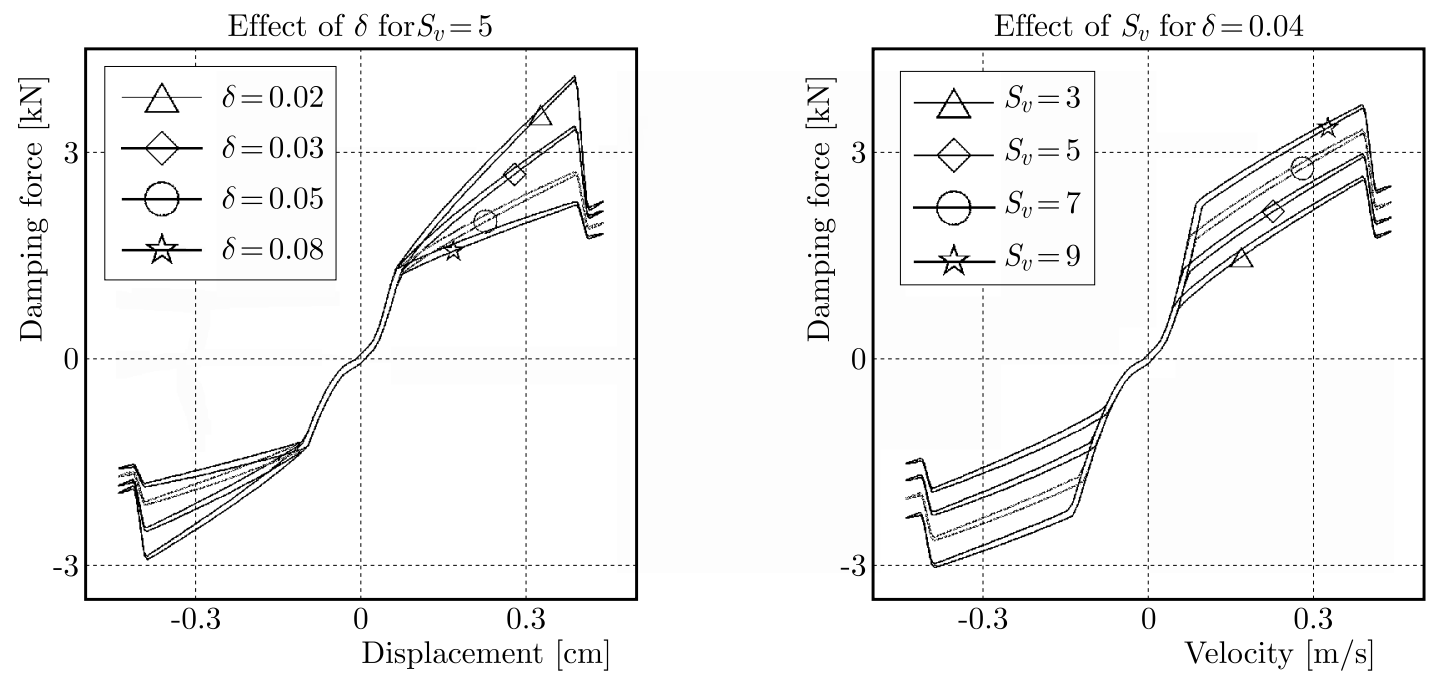

Fig. 8. Influence of the parameters $\delta$ and $S_{v}$ on the force characteristics $(f=1.4 \mathrm{~Hz}, a=5 \mathrm{~cm}$, $\left.h=2 \mathrm{~cm}, \alpha=0.004, \gamma=0.012, k_{p}=2, k_{b}=0.5\right)$

The dimensionless parameter $\delta\left(\delta_{2-1}=\delta, \delta_{1-2}=0.8 \delta\right)$ determines the maximum areas of the orifices controlled by the pressure difference. It influences the characteristic in the higher velocities range (Fig. 8). When the piston velocity increases, the pressure difference in chambers $K_{1}$ and $K_{2}$ also increases and, in consequence, the valves in the piston are opening and the damping force decreases. For larger values of the parameter $\delta$, in the compression as well as in the rebound process, the inclination of curves determining the damping force dependence on 
velocity decreases. For high piston velocity near the piston zero position, the damping force of the shock absorber abruptly changes. The reason of this sudden change constitutes unblocking (or blocking) of flows through the bypass, which entails a decrease (or increase) in the pressure difference in the neighbouring chambers. The dimensionless parameter $S_{v}$ characterises the preload force and decides about the location of the characteristic inflection point. This means that the point in which the inclination angle of curves changes (Fig. 8). When the parameter $S_{v}$ changes, the characteristic shape related to opening of the orifices changes for higher velocities $\dot{x}$. Simultaneously, the maximum values of the damping force increase.

The damper performance within the range of large relative displacements of the piston depends additionally on parameters characterising the bypass, i.e. on the parameter $\gamma$ $\left(\gamma_{2-1}=\gamma_{1-2}=\gamma\right)$ (determining the orifice cross-section area), on distance $h$ (it is assumed that $h_{1}=h_{2}=h$ ) determining placement of bypass openings in the working cylinder, and on the parameter $k_{b}$ (characterising elastic properties of the valve).
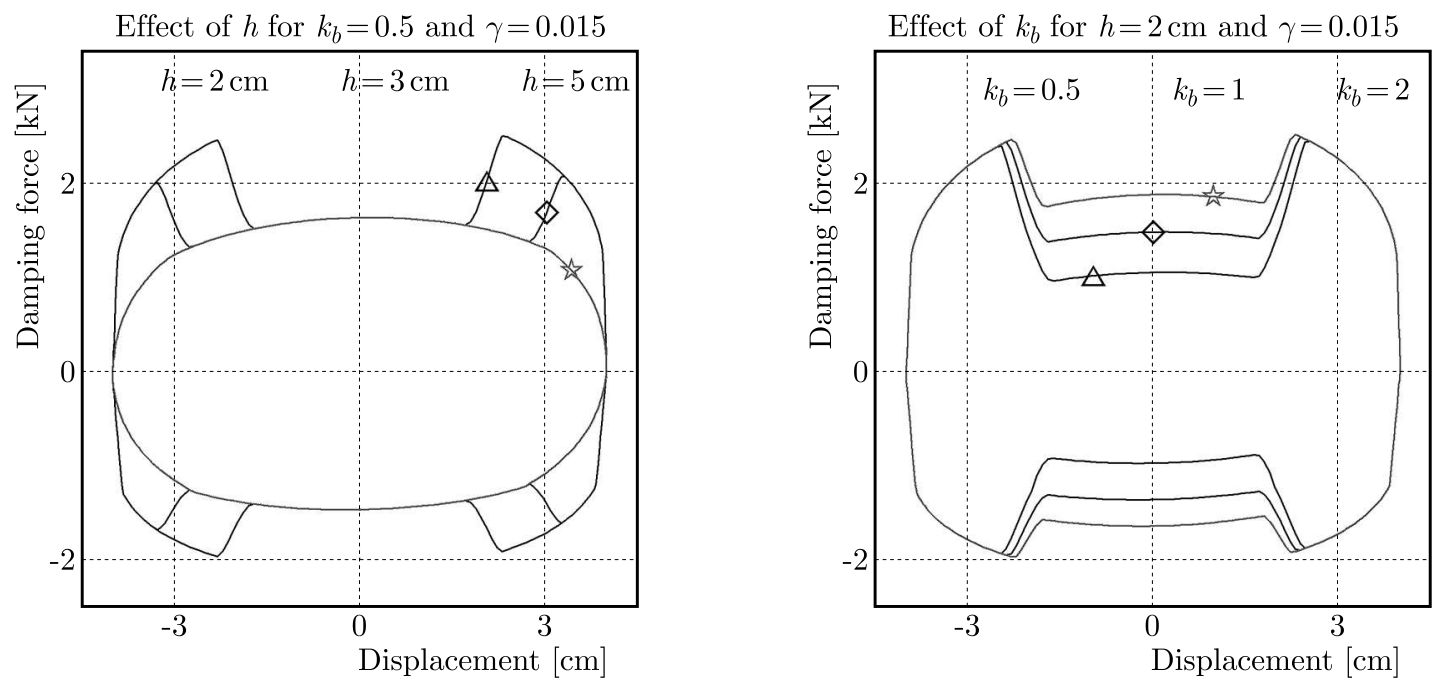

Fig. 9. Influence of the parameters $h$ and $k_{b}$ on the force characteristics $(f=1.4 \mathrm{~Hz}, a=4 \mathrm{~cm}$, $\beta=0.004, \beta=0.04, k_{p}=2$ )

Figure 9 shows the influences of the parameters $h$ and $k_{b}$ on the damping force as a function of piston displacements. The sudden change of the damping force occurs in two ranges of the displacements: $-h-r<x<-h+r$ and $h-r<x<h+r$. In these both ranges the area change is given by equation (2.24).

Since for higher values of the parameter $h$ the oil flow through the bypass can be blocked only in the case of large piston displacements, the shock absorber in a wide range of vibration amplitudes behaves in a similar fashion as the classic shock absorber without the bypass (curve for $h=5 \mathrm{~cm}$ ). The change of the characteristic occurs only when the amplitude exceeds a certain limited value, dependent on the parameter $h$. Along with a decrease of $k_{b}$, the damping force decreases significantly in the range of small displacements. For higher values of the parameter $k_{b}$, the bypass valve is not fully open, that is to say the effective area $A$ bypass is smaller.

Summing up the above conclusions, the application of more stiff valves causes a decrease of the force step change (improves safety of the ride), however, it occurs at the expense of increasing damping forces within the range of small displacements (disadvantageous for the ride comfort). In some (currently produced) shock absorbers, the user can independently change the parameters of the bypass valve (parameter $k_{b}$ ), i.e. can adjust the damper characteristic for the conditions of the ride. 


\section{Conclusions}

The non-linear model describing behaviour of the mono-tube shock absorber with a bypass, applied in passive systems of car suspensions, is proposed in this paper. Controlling of the oil flow between the damper chambers depending on the present pressure as well as on relative displacements of the piston is considered. A change in the oil bulk modulus caused by the pressure change is taken into account, and a relevant description allowing for efficient numerical simulations is provided. The developed model allows one to investigate the most important properties of mono-tube hydraulic shock absorbers in a wide range of amplitudes and excitation frequencies.

Several numerical simulations have been performed and their most important results are presented in this work. The influence of structural parameters of the shock absorber on the damping force characteristics is investigated in detail. Quantitative analysis indicates a significant influence of parameters depending on geometrical and physical properties of structural elements of valves in the main piston and in the bypass of the shock absorber. Although the results obtained are qualitatively consistent with the results of other authors, it is advisable to experimentally verify the assumptions used in the modeling process.

The application of bypasses allows one to obtain satisfactorily high damping forces for large relative displacements of the piston. In such a case, the shock absorber meets the requirements of a 'hard' damper, improving by that safety of the ride. When the relative displacements are small, the bypass is active and a 'soft' damper characteristic provides a high comfort level of the ride.

The global analysis of the efficiency of application of bypasses to vehicle shock absorbers requires development of a vehicle model with the tested damper and investigation of the influence various types of excitations on indices defining the comfort, (e.g. max or RMS value of velocity of the so-called spring-supported mass) as well as safety of the ride (e.g. dynamic component of the reaction exerted on the wheel of the vehicle). Determining such system responses for a sinusoidal signal with a variable frequency (e.g. sweep type), impulse or random signals allow one to better understand the shock absorber performance in various situations.

\section{References}

1. Alonso M., Comas Á., 2006, Modelling a twin tube cavitating shock absorber, Proceedings of the Institution of Mechanical Engineers, Part D: Journal of Automobile Engineering, 220, 8, 1031-1040

2. Сно B.H., LeE H.W., Он J.S., 2002, Estimation technique of air content in automatic transmission fluid by measuring effective bulk modulus, International Journal of Automotive Technology, $\mathbf{3}, 2,57-61$

3. Czop P., SŁawik D., 2011, A high-frequency first-principle model of a shock absorber and servo-hydraulic tester, Mechanical Systems and Signal Processing, 25, 6, 1937-1955

4. Farjoud A., Ahmadian M., Craft M., Burke W., 2012, Nonlinear modeling and experimental characterization of hydraulic dampers: effects of shim stack and orifice parameters on damper performance, Nonlinear Dynamics, 67, 2, 1437-1456

5. Ferdek U., Łuczko J., 2012, Modeling and analysis of a twin-tube hydraulic shock absorber, Journal of Theoretical and Applied Mechanics, 50, 2, 627-638

6. Ferdek U., Łuczko J., 2015, Performance comparison of active and semi-active SMC and LQR regulators in a quarter-car model, Journal of Theoretical and Applied Mechanics, 53, 4, 811-822

7. Ferdek U., ŁucZKo J., 2016, Vibration analysis of a half-car model with semi-active damping, Journal of Theoretical and Applied Mechanics, 54, 2, 321-332 
8. GoŁdasz J., 2015, Theoretical study of a twin-tube magnetorheological damper concept, Journal of Theoretical and Applied Mechanics, 53, 4, 885-894

9. KIng L., 2014, Adjustable internal bypass shock absorber featuring a fluid flow regulator, U.S. Patent No. 8,820,495

10. Lee C.T., Moon B.Y., 2006, Simulation and experimental validation of vehicle dynamic characteristics for displacement-sensitive shock absorber using fluid-flow modelling, Mechanical Systems and Signal Processing, 20, 2, 373-388

11. Manring N.D., 1997, The effective fluid bulk-modulus within a hydrostatic transmission, Journal of Dynamic Systems, Measurement, and Control, 119, 3, 462-466

12. Marking J., 2014, Bypass for a suspension damper, U.S. Patent No. 8,627,932

13. MerritT H.E., 1967, Hydraulic Control Systems, John Wiley \& Sons

14. NorgaArd B.M., Cimins D.J., 2009, Fluid flow regulation of a vehicle shock absorber/damper, U.S. Patent No. 7,628,259

15. Purdy D.J., 2000, Theoretical and experimental investigation into an adjustable automotive damper, Proceedings of the Institution of Mechanical Engineers, Part D: Journal of Automobile Engineering, 214, 265-283

16. Ramos J.C., Rivas A., Biera J., Sacramento G., Sala J.A., 2005, Development of a thermal model for automotive twin-tube shock absorbers, Applied Thermal Engineering, 25, 11, 1836-1853

17. SAPiński B., Rosó Ł M., 2007, MR damper performance for shock isolation, Journal of Theoretical and Applied Mechanics, 45, 1, 133-145

18. Silveira M., Pontes B.R., Balthazar J.M., 2014, Use of nonlinear asymmetrical shock absorber to improve comfort on passenger vehicles, Journal of Sound and Vibration, 333, 7, 2114-2129

19. Talbott M.S., Starkey J., 2002, An experimentally validated physical model of a high-performance mono-tube damper, SAE Technical Paper

20. Titurus B., Du Bois J., Lieven N., Hansford R., 2010, A method for the identification of hydraulic damper characteristics from steady velocity inputs, Mechanical Systems and Signal Processing, 24, 8, 2868-2887

21. VAN DE VEN J.D., 2013, On fluid compressibility in switch-mode hydraulic circuits - Part I: Modeling and analysis, Journal of Dynamic Systems, Measurement and Control, 135, 2, 021013 